\title{
Renal Phenotype in Mitochondrial Diseases: A Multicenter Study
}

\author{
Maria Parasyria Per Brandström ${ }^{a, b}$ Johanna Uusimaac, d Elsebet Ostergaard ${ }^{\mathrm{e}}$ \\ Omar Hikmat $^{f, g}$ Pirjo Isohanni ${ }^{\text {h, }}$ i Karin Naess ${ }^{j}$ I.F.M. de Coo ${ }^{k}$ \\ Andrés Nascimento Osoriol Matti Nuutinen ${ }^{c, d} \quad$ Christopher Lindberg ${ }^{m}$ \\ Laurence A. Bindoffg, n Már Tulinius ${ }^{a, b}$ Niklas Darin ${ }^{a, b} \quad K^{b}$ alliopi Sofou ${ }^{a, b}$
}

aDepartment of Paediatrics, Region Västra Götaland, Sahlgrenska University Hospital, Gothenburg, Sweden;

${ }^{b}$ Department of Paediatrics, Institute of Clinical Sciences, Sahlgrenska Academy, University of Gothenburg, Gothenburg, Sweden; ' Department of Children and Adolescents, Oulu University Hospital, Oulu, Finland; ${ }^{\text {dPEDEGO }}$ Research Unit, Research Unit for Pediatrics, Pediatric Neurology, Pediatric Surgery, Child Psychiatry, Dermatology, Clinical Genetics, Obstetrics and Gynecology, Ophthalmology, Otorhinolaryngology, Medical Research Center Oulu (MRC Oulu), University of Oulu, Oulu, Finland; 'Department of Clinical Genetics, Copenhagen University Hospital Rigshospitalet, Copenhagen, Denmark; fDepartment of Pediatrics and Adolescent Medicine, Haukeland University Hospital, Bergen, Norway; ${ }^{9}$ Department of Clinical Medicine (K1), University of Bergen, Bergen, Norway; hDepartment of Pediatric Neurology, Children's Hospital, University of Helsinki and Helsinki University Hospital, Helsinki, Finland; 'Research Programs Unit, Stem Cells and Metabolism, University of Helsinki, Helsinki, Finland; jCentre for Inherited Metabolic Diseases, Karolinska University Hospital, Stockholm, Sweden; ${ }^{\text {kDepartment }}$ Toxicogenomics, Faculty of Health, Medicine and Life Sciences, Graduate School MHeNS, Maastricht University, Maastricht, The Netherlands; 'Neuromuscular Diseases Unit, Hospital Sant Joan de Déu, Barcelona, Spain; mDepartment of Neurology, Neuromuscular Centre, Sahlgrenska University Hospital, Gothenburg, Sweden; ${ }^{\mathrm{n} N e u r o-}$ SysMed, Center of Excellence for Clinical Research in Neurological Diseases, Haukeland University Hospital, Bergen, Norway

\section{Keywords}

Mitochondrial disease · Acute kidney injury · Mitochondrial DNA $\cdot$ Renal manifestations

\begin{abstract}
Aims: This study aimed to investigate associations between renal and extrarenal manifestations of mitochondrial diseases and their natural history as well as predictors of renal disease severity and overall disease outcome. The secondary aim was to generate a protocol of presymptomatic assessment and monitoring of renal function in patients with a defined mitochondrial disease. Methods: A multicenter, retro-
\end{abstract}

spective cohort study was performed by the Mitochondrial Clinical and Research Network (MCRN). Patients of any age with renal manifestations associated with a genetically verified mitochondrial disease were included from 8 expert European centers specializing in mitochondrial diseases: Gothenburg, Oulu, Copenhagen, Bergen, Helsinki, Stockholm, Rotterdam, and Barcelona. Results: Of the 36 patients included, two-thirds had mitochondrial DNA-associated disease. Renal manifestations were the first sign of mitochondrial disease in 19\%, and renal involvement was first identified by laboratory tests in $57 \%$ of patients. Acute kidney injury occurred in 19\% of patients and was the first sign of renal disease in the majority of these. The most common re- karger@karger.com www.karger.com/kdd

Karger $\stackrel{\text { ' }}{5}$
(C) 2022 The Author(s)

Published by S. Karger AG, Basel

This is an Open Access article licensed under the Creative Common Attribution-NonCommercial-4.0 International License (CC BY-NC) (http://www.karger.com/Services/OpenAccessLicense), applicable to the online version of the article only. Usage and distribution for commercial purposes requires written permission.
Correspondence to:

Kalliopi Sofou, kalliopi.sofou@vgregion.se 
nal manifestation was chronic kidney disease $(75 \%$ with stage 2 or greater), followed by tubulopathy $(44.4 \%)$, the latter seen mostly among patients with single large-scale mitochondrial DNA deletions. Acute kidney injury and tubulopathy correlated with worse survival outcome. The most common findings on renal imaging were increased echogenicity and renal dysplasia/hypoplasia. Renal histology revealed focal segmental glomerulosclerosis, nephrocalcinosis, and nephronophthisis. Conclusion: Acute kidney injury is a distinct renal phenotype in patients with mitochondrial disease. Our results highlight the importance to recognize renal disease as a sign of an underlying mitochondrial disease. Acute kidney injury and tubulopathy are 2 distinct indicators of poor survival in patients with mitochondrial diseases.

(c) 2022 The Author(s).

Published by S. Karger AG, Basel

\section{Introduction}

Mitochondrial diseases are a heterogeneous group of disorders caused by defects in the mitochondrial oxidative phosphorylation system. Multiple organs can be affected, especially those with high energy requirements, such as the central nervous system, skeletal muscle, heart, kidneys, and liver [1]. Renal dysfunction may be the presenting feature of mitochondrial diseases, but is most commonly seen after the onset of neurological manifestations, often as part of a multisystem phenotype [2]. The reported prevalence of renal involvement in mitochondrial diseases varies from 25 to $50 \%$ [3-5], and the underlying genetic etiology seems to play a role both in the varying prevalence and type of renal manifestations [3].

Reported renal manifestations of mitochondrial diseases include tubular dysfunction, interstitial nephritis, nephrotic syndrome, cystic disease, and end-stage renal failure $[2,3,6,7]$. Proximal tubulopathy is the commonest phenotype in childhood-onset mitochondrial disease and can be associated with both nuclear DNA (nDNA) variants and large-scale mitochondrial DNA (mtDNA) deletions $[6,8]$. Apparently, the high energy requirements and the inability to synthesize adenosine triphosphate anaerobically from glycolysis make proximal tubular cells extremely vulnerable to energy crisis secondary to mitochondrial dysfunction [9].

Severe tubular dysfunction, known as de Toni-DebréFanconi syndrome, occurs in patients with mtDNA deletion syndromes such as Kearns-Sayre and Pearson syndrome [6]. This syndrome is characterized by renal tubular acidosis, hyperphosphaturia, glycosuria, and generalized aminoaciduria with low-molecular-weight proteinuria $[3,6]$. mtDNA point mutations such as m.3243A $>$ G that cause mitochondrial encephalomyopathy, lactic acidosis and stroke-like episodes, and maternally inherited diabetes and deafness have been mostly associated with phenotypes such as tubulointerstitial nephritis, focal segmental glomerulosclerosis, and progressive renal failure $[8,10]$. The renal phenotype of nDNAassociated mitochondrial disease is more diverse [8].

As mitochondrial diseases are often multisystemic and associated with severe neurological phenotypes, renal manifestations can go undetected until the renal symptoms are severe, or the patient has developed end-stage renal failure. Recognizing an underlying mitochondrial disease when renal symptoms occur first or alone can be equally difficult. Currently, there are few cohort studies available to inform our practice [3-5], the majority being clinical case reports and reviews. Therefore, we undertook this multinational cohort study to investigate associations between renal and extrarenal onset of mitochondrial disease, the natural history of renal manifestations, and predictors of renal disease severity and overall disease outcome. On the basis of our results, we propose a protocol for the assessment and monitoring of renal function in presymptomatic patients with known mitochondrial disease.

\section{Methods}

\section{Patients}

This multicenter, retrospective cohort study supported by the Mitochondrial Clinical and Research Network (MCRN) included patients from 8 expert European centers specializing in mitochondrial diseases: Gothenburg, Oulu, Copenhagen, Bergen, Helsinki, Stockholm, Rotterdam, and Barcelona. Patients of any age with a genetically defined mitochondrial disease with renal manifestations were included.

\section{Data Collection}

Data were collected using a standardized case report form (available upon request). Collected data included results from genetic testing, clinical, biochemical, morphological, and imaging assessments, comorbidities, and treatment used. As this was a retrospective study, a cutoff for missing data of $10 \%$ or less was implemented to ensure quality of statistical inferences. Assessments for 5 patients have been included in other publications, as detailed in Tables 1 and 2.

\section{Clinical and Laboratory Variables}

Occurrence and severity of renal disease was evaluated by estimating the amount of proteinuria and/or hematuria and by measuring the glomerular filtration rate (GFR) either directly by EDTA-cleareance (in $\mathrm{mL} / \mathrm{min} / 1.73 \mathrm{~m}^{2}$ ) or by calculating the estimat- 


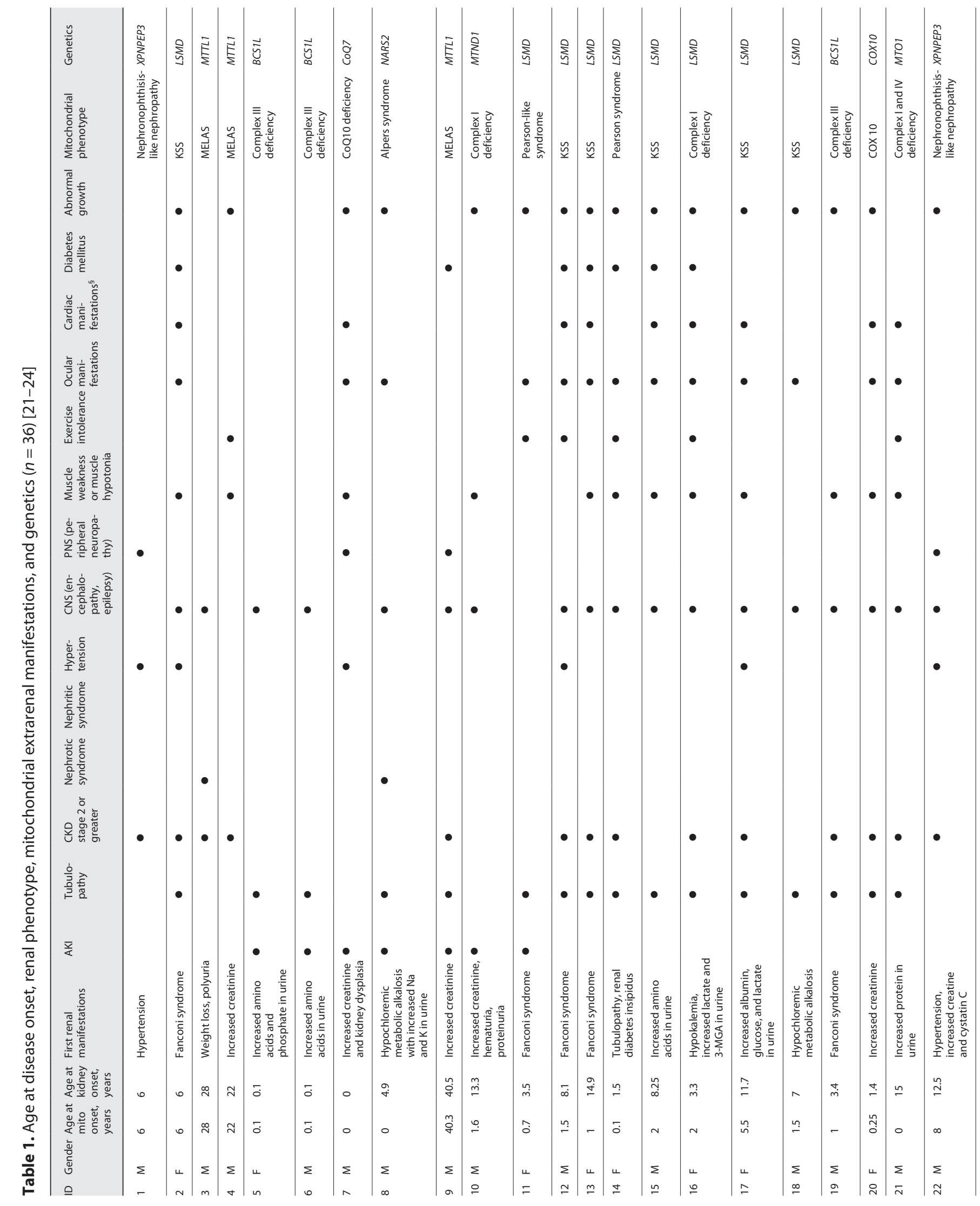




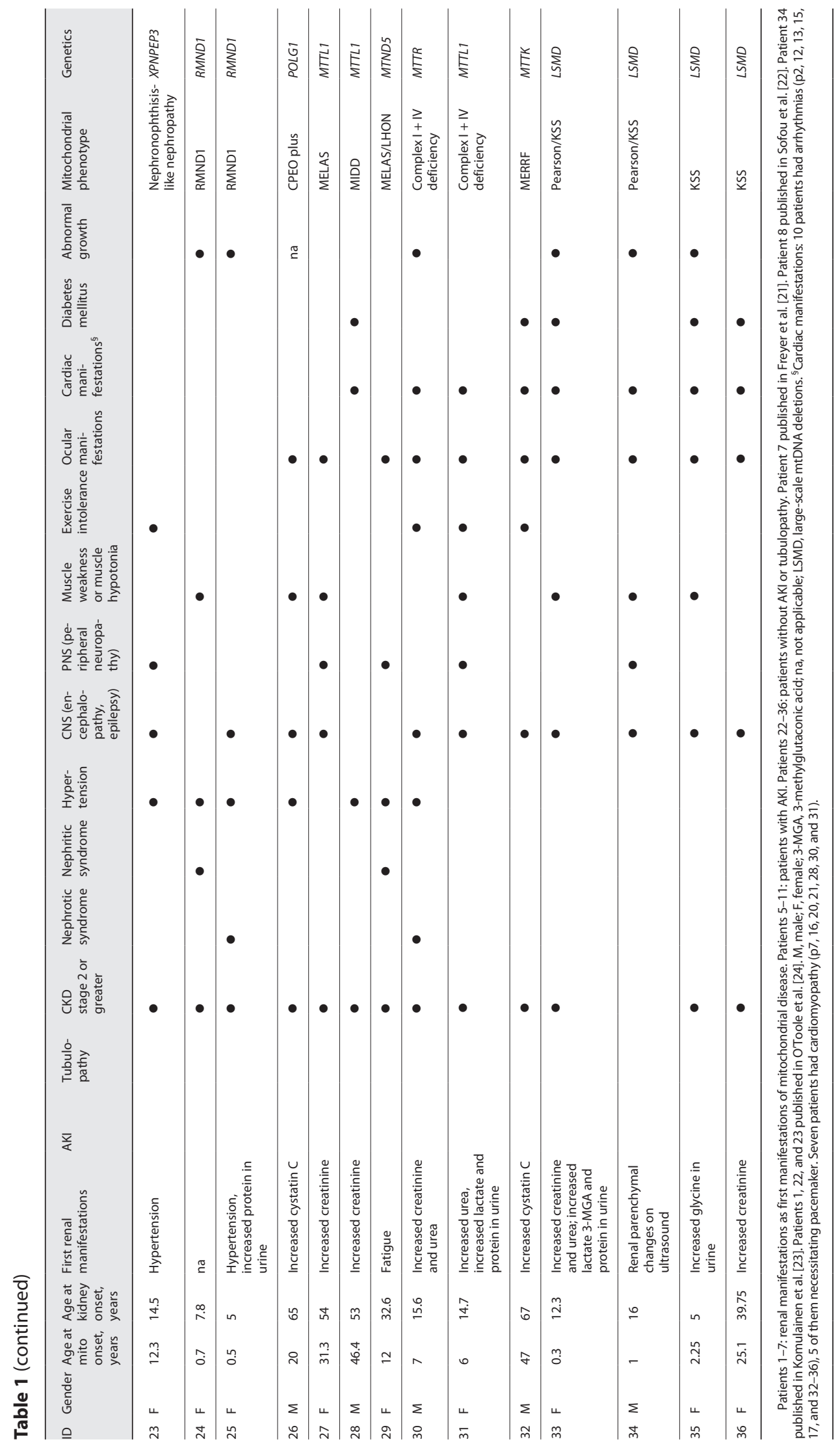




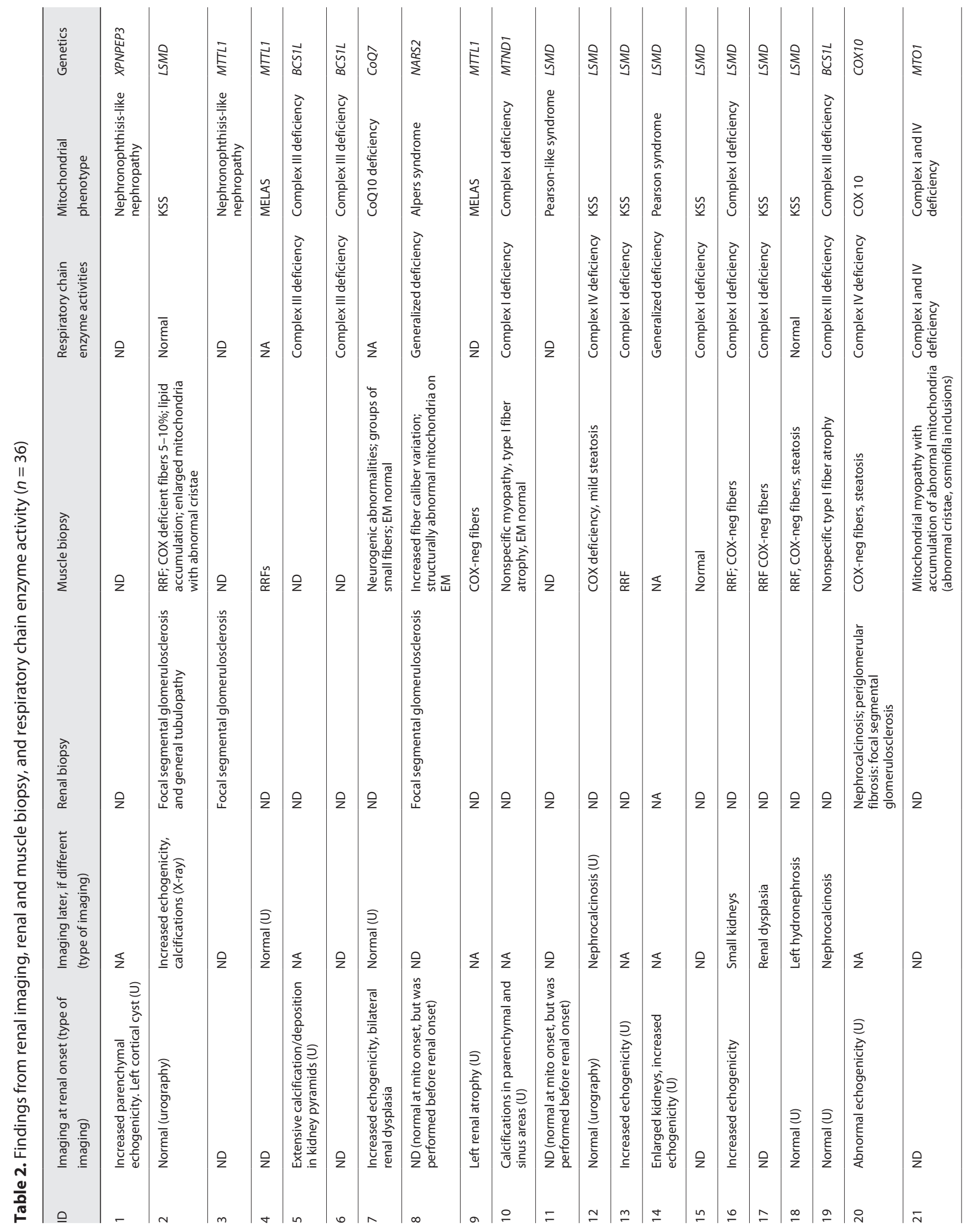




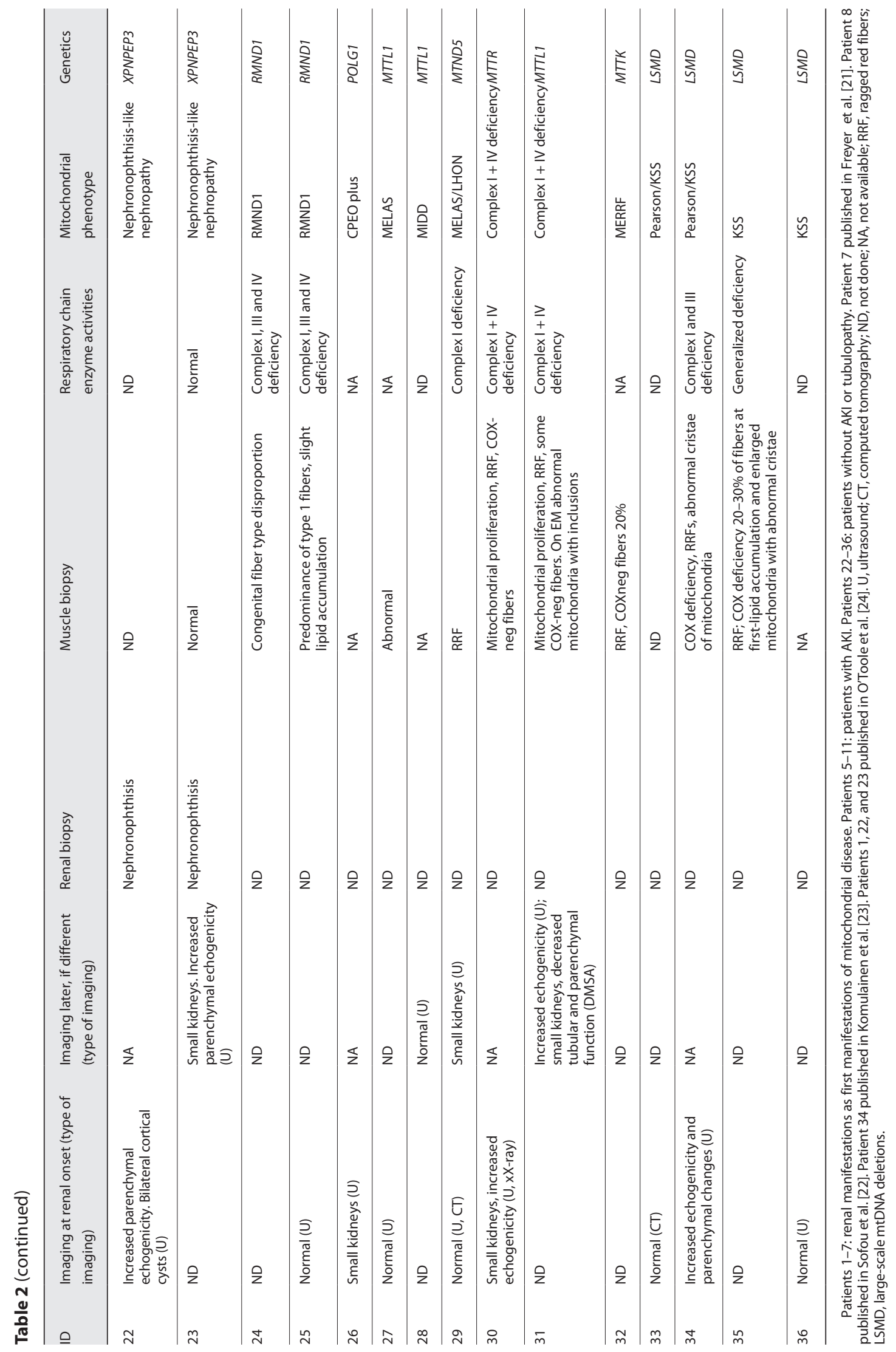


ed GFR (eGFR) using the creatinine-based bedside Schwartz equation [11]. For defining and staging chronic kidney disease (CKD), we used the Kidney Disease Improving Global Outcomes (KDIGO) guidelines [12]. CKD stage 2 or greater was defined as a GFR $<90 \mathrm{~mL} / \mathrm{min} / 1.73 \mathrm{~m}^{2}$. Acute kidney injury (AKI) was diagnosed clinically, relying on changes in serum creatinine and/or urine output, as proposed by KDIGO [13]. Increased excretion of albumin and total protein in urine was evaluated based on the KDIGO guidelines [13].

Nephrotic syndrome was defined as severe albuminuria (urine albumin/creatinine ratio $>400 \mathrm{mg} / \mathrm{mmol}$ [or $>3500 \mathrm{mg} / \mathrm{g}$ ] or urine albumin $>50 \mathrm{mg} / \mathrm{kg} / 24 \mathrm{~h}$ ), hypoalbuminemia (serum albumin $<25$ $\mathrm{g} / \mathrm{L}$ ), and edema. For the diagnosis of nephritic syndrome, the patient had to fulfill all of the following: (a) hematuria ( $\geq 2+$ on urine dipstick analysis) with or without proteinuria, (b) increased serum creatinine adjusted for age, and (c) hypertension (systolic or diastolic arterial pressure $\geq 95$ th percentile adjusted for age and height).

Proximal tubulopathy, i.e., dysfunction of the renal proximal tubules, was defined as having one or more of the following: lowmolecular-weight proteinuria, general aminoaciduria, glucosuria (with normal plasma glucose levels), bicarbonate loss resulting in renal tubular acidosis, and electrolyte wasting. The combination of bicarbonate loss with variable renal wasting of amino acids, glucose, phosphate, uric acid, and other solutes was defined as renal Fanconi syndrome. Renal Fanconi syndrome with hypophosphatemic rickets and glycosuria was defined as de Toni-Debré-Fanconi syndrome [14]. Isolated distal tubulopathy was defined as urinary wasting of electrolytes $\left(\mathrm{Na}^{+}, \mathrm{K}^{+}\right.$, and $\left.\mathrm{Cl}^{-}\right)$in the absence of signs of proximal tubular dysfunction, while coexistence of proximal and distal tubulopathy was characterized as generalized tubulopathy.

\section{Protocol Development}

We developed a protocol for the assessment and monitoring of renal function in mitochondrial disease patients prior to established kidney disease. Development of this protocol relied on results from this study and the consensus for patient care standards [15] and was critically reviewed by all MCRN investigators who participated in this study.

\section{Statistical Considerations}

The statistical evaluations performed were mainly exploratory. Survival outcomes were estimated with the help of Kaplan-Meier analysis. The $\chi^{2}$ test was used to test the association between categorical variables. The independent $t$ test was used to test differences between categorical variables, such as gender, and continuous variables, such as age at onset. All statistical tests were 2 -sided and performed at a 0.05 significance level.

\section{Results}

A total of 36 patients were included (19 males and 17 females). The majority had mtDNA-associated disease $(23 / 36)$. The median age at onset of mitochondrial disease was 2 years (prenatal-47 years of age). Patients were followed for a median period of 15 years (0.1-49.5 years).

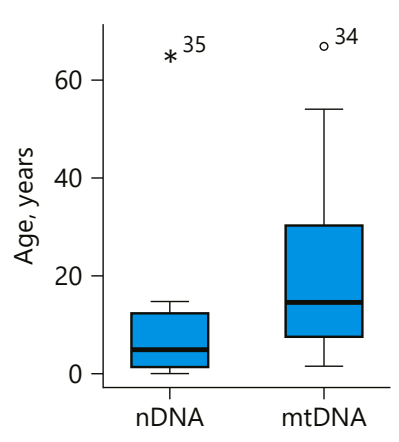

\begin{tabular}{|l|l|}
\hline nDNA & mIDNA \\
\hline XPNPEP3 (3) & LSMD (13) \\
\hline BCS1L (3) & MTND1 (1) \\
\hline CoQ7 (1) & MTND5 (1) \\
\hline COX10 (1) & MTTL1 (6) \\
\hline MTO1 (1) & MTTR (1) \\
\hline RMND1 (2) & MTTK (1) \\
\hline POLG1 (1) & \\
\hline NARS2 (1) & \\
\hline
\end{tabular}

$\mathrm{nDNA}=$ nuclear DNA; mtDNA = mitochondrial DNA; LSMD: large-scale mtDNA deletions

Fig. 1. Age at renal disease onset in patients with mtDNA versus nDNA-associated disease ( $p=0.062$ for the entire group; $p=0.011$ when excluding the 2 extreme values of ID 34 [=67yo] and ID 35 $[=65 \mathrm{yo}])$. A list of underlying genetics is presented with the corresponding number of patients in parenthesis. LSMD, large-scale mtDNA deletions.

\section{Onset of Renal Manifestations}

The median age at onset of renal manifestations was 12 years (birth-67 years). Renal manifestations occurred earlier in patients with nDNA-associated mitochondrial disease (nDNA 5 y; mtDNA 15 y; $p=0.011$; Fig. 1). A summary of patients' age at disease onset, renal phenotype, mitochondrial extrarenal manifestations, and genotype is shown in Table 1.

Renal manifestations were the first sign of mitochondrial disease (ID1-7 in Table 1) in 7 patients, either alone (ID1, 4, and 5) or in combination with anemia (ID2), hearing impairment (ID3), or epilepsy (ID6). One patient had multiorgan disease at birth, which, apart from renal manifestations, included lung hypoplasia, persistent pulmonary hypertension, systemic arterial hypertension, and secondary left ventricle hypertrophy (ID7). Three of these 7 patients presented with AKI in the first month of life. In the patient with multiorgan involvement (ID7), renal manifestations resolved spontaneously by the first year of life and had not reappeared by the last follow-up visit at 11 years of age.

Renal involvement was first captured by laboratory tests, either as part of routine workup or fortuitously, in 20 patients $(20 / 35$; 1 unknown) (Table 1$)$. Isolated increased levels of serum creatinine or cystatin $C$ were the presenting feature in 9 patients $(9 / 35 ; 1$ unknown).

Fifteen patients presented with symptoms such as fatigue, polydipsia, polyuria, and hypertension, either alone 
Fig. 2. Age when the first renal manifestations occurred, grouped by type of renal manifestations ( $n=34 / 36$; 1 with abnormal renal imaging and 1 unknown), i.e., abnormal renal function tests in blood alone $(n=$ $10)$, abnormal renal function tests in urine alone $(n=6)$, abnormal renal function tests in blood and urine without clinical signs $(n$ $=4)$, arterial hypertension alone $(n=4)$, Fanconi syndrome $(n=5)$, and clinical signs or symptoms of renal disease with or without arterial hypertension $(n=5)$.

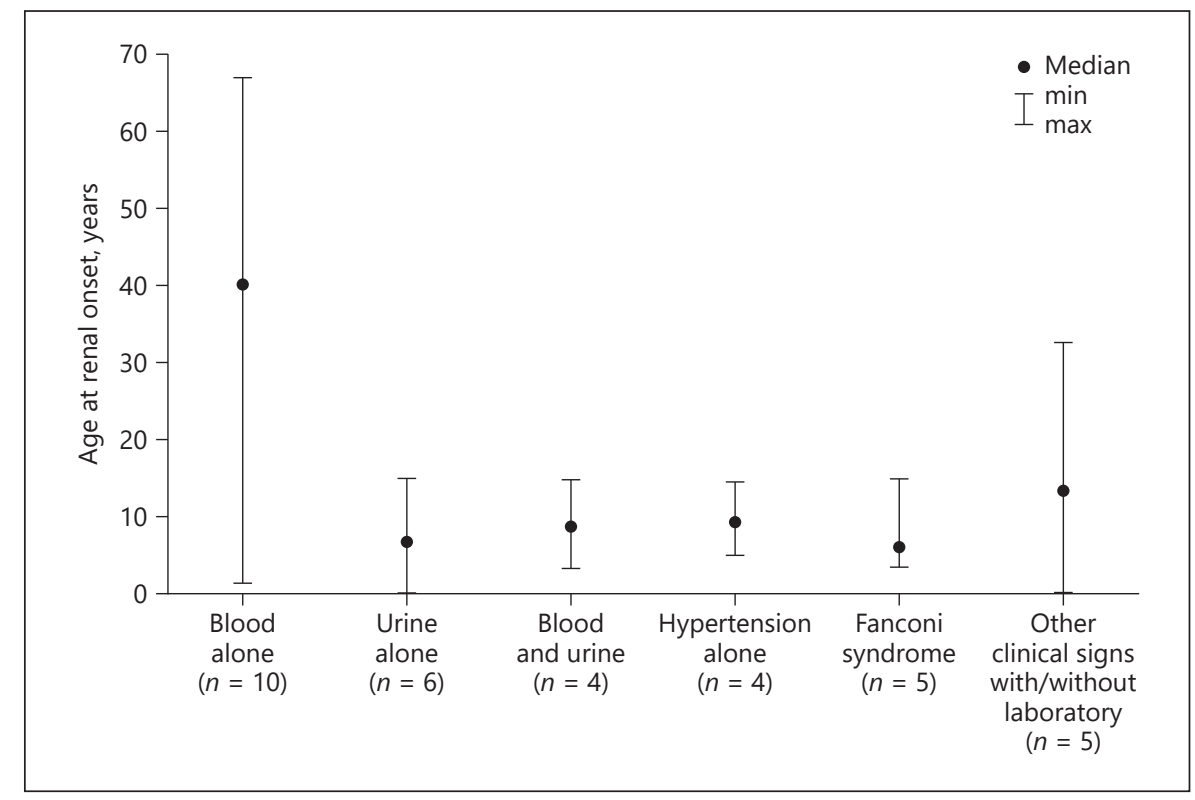

or in combination with abnormal laboratory tests, and/or abnormal renal imaging. Fanconi syndrome was the presenting feature in 5 patients $(5 / 35 ; 1$ unknown).

The identification of renal disease on routine laboratory testing or fortuitously occurred in all age groups and, as expected, tended to occur later than in those with clinical signs of renal disease ( $p=0.108)$ (Fig. 2). No correlation was found between the type of presenting renal manifestations and gender, genetic background, or renal disease outcome.

\section{Acute Kidney Injury}

Seven of 36 patients developed AKI (ID5-11, Table 1) at a median age of 4 years (birth-46 years), and in 5, AKI was the first manifestation of renal disease. CKD preceded AKI in 1 patient (ID9). No repeated episodes of AKI were seen in any of our patients. The occurrence of tubulopathy was common among patients with a history of AKI (5/7). Four of 7 patients developed Fanconi syndrome, of whom one fulfilled the criteria for de ToniDebré-Fanconi syndrome (ID11). No correlation was found between the history of AKI and the gender, age at onset of renal disease, or mitochondrial genetics. Rhabdomyolysis was not seen in any of the patients.

\section{Other Renal Manifestations, Laboratory Findings, and Management}

CKD with decreased GFR (stage 2 or greater) was found in 27 of 36 patients, as shown in Table 1. Twentytwo patients had a CKD stage 3 to 5 by the time of last follow-up (median age 26.35 years old, min-max: 5.7569.5). The incidence of CKD was equally distributed across gender, age at renal onset, and genetic background. Seven patients underwent renal replacement therapy, i.e., dialysis (4) and/or renal transplantation (3). The age at start of dialysis was 19 years (min-max: 18-20 years; 1 unknown). Dialysis was initiated at a median of 13 years after onset of renal manifestations (min-max: 5-16 years; 1 unknown). One of the 4 patients with dialysis received a renal transplant shortly afterward, while another patient died 2 months after initiation of dialysis. One patient was on dialysis for 3 years, until death due to cardiac complications.

Tubulopathy was found in 16 patients (16/36): Fanconi syndrome (7/16), proximal tubulopathy $(4 / 16)$, generalized tubulopathy (1/16), and unspecified (4/16). Tubulopathy was significantly more common among patients with large-scale mtDNA deletions, compared to patients with other genetic etiologies $(p=0.025)$.

Hyperlactatemia was found at least once in 27 patients (27/32; unknown in 4 patients). An increased excretion of lactate and/or 3-methylglutaconic acid in urine was common (13/18), while multiple organic acidurias were seen in 3 patients. General aminoaciduria was seen in 10 of 24 patients, while 2 patients, both with KSS due to large-scale mtDNA deletions, had repeatedly increased excretion of glycine in urine (ID18 and 35). Increased excretion of electrolytes in urine and/or low plasma levels necessitating electrolyte supplementation was found in 17 patients $(17 / 36)$. 


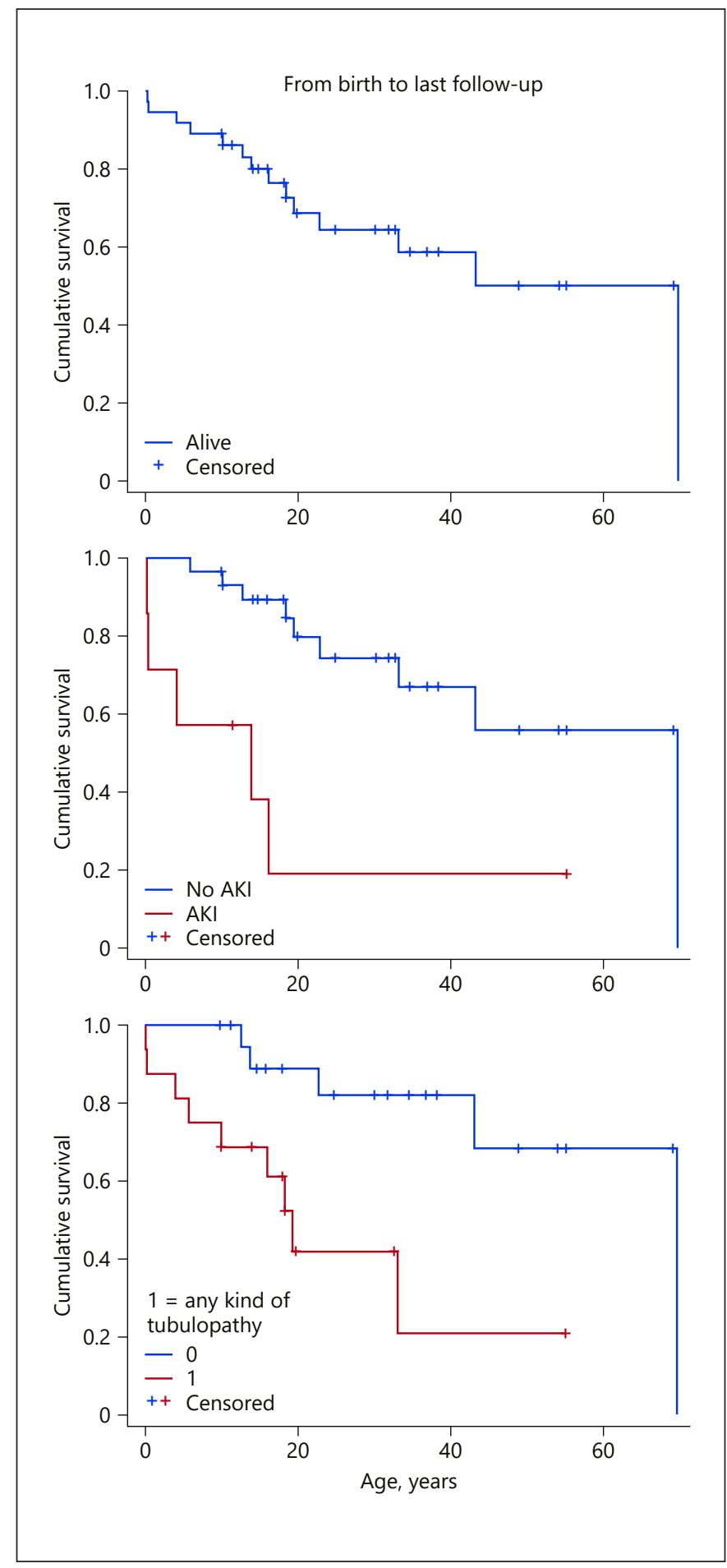

Fig. 3. Survival curves for the entire cohort ( $n=36$; a), acute kidney injury $(n=7 / 36 ; \mathbf{b})$ versus the rest of the cohort, and any kind of tubulopathy ( $n=16 / 36$; c) versus the rest of the cohort.
Hypertension was found in 12 patients $(12 / 36)$ and was the presenting feature in 4 of them; i.e., in 3 XPNPEPpatients (ID1, 22, and 23) and in 1 RMND1-patient (ID25). Nephrotic syndrome was found in 4 patients and nephritic syndrome in 2 patients (Table 1).

\section{Renal Imaging and Biopsy Findings}

Renal imaging was performed in 29 patients (29/36) and was abnormal in 21 . In 5 of 21 , renal imaging was initially normal. Table 2 summarizes the renal imaging and biopsy findings, along with the results from the respiratory chain enzyme analyses. The most common findings on renal imaging were increased echogenicity (12/21), renal dysplasia/hypoplasia (9/21), calcifications/ nephrocalcinosis (5/21), and renal cysts (2/21). Renal biopsy was performed in 6 patients and was abnormal in all. Main renal biopsy findings were focal segmental glomerulosclerosis, nephrocalcinosis, and nephronophthisis (Table 2).

\section{Survival Outcome}

Fourteen of 36 patients died at a median age of 15 years (0.1-69.5 years) (Fig. 3). A history of AKI or tubulopathy correlated with reduced survival $(p=0.002$ and $p=0.005$, respectively) (Fig. 3). No other specific renal phenotype correlated with patients' survival outcomes, nor was there any correlation to the underlying type of genetic defect (mtDNA vs. nDNA-associated disease, $p=0.568$ ).

In our study, 5 of 7 patients with AKI died at a median age of 4 years ( $0.1-13.75$ years), 4 of them within 6 months after the onset of AKI. None of these patients suffered from cardiovascular, pulmonary, or endocrinological manifestations other than abnormal growth.

\section{Discussion}

Renal function is highly dependent on aerobic respiration, making it vulnerable to energy deprivation. Defects in mitochondrial energy metabolism have been associated with various renal manifestations, including proximal tubulopathy and CKD. Our results emphasize the occurrence of AKI as a distinct renal phenotype in patients with mitochondrial disease. We further elaborate on clinical indicators of isolated renal disease suggestive of underlying mitochondrial disease.

AKI is characterized by an abrupt decrease in the GFR. Mitochondrial damage including mitochondrial fragmentation, swelling, and loss of inner structure contribute critically to AKI development [16]. Mitochondrial 
Table 3. Protocol for the assessment and monitoring of renal function in mitochondrial disease patients without known renal involvement (presymptomatic monitoring)

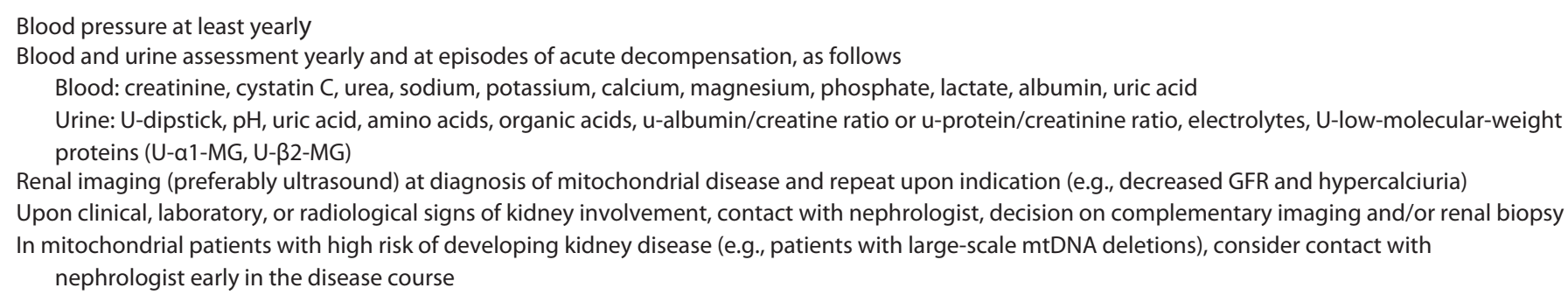

U-a1-MG, urine alpha-1-microglobulin; U- $\beta 2-M G$, urine beta-2-microglobulin.

oxidative stress and decreased adenosine triphosphate production primarily affect the renal tubular cells in AKI [16]. Mitophagy and mitochondrial biogenesis are 2 renoprotective mechanisms with a substantial role in the recovery phase of AKI $[16,17]$. If these mechanisms are compromised, as in patients with genetic mitochondrial disease, the kidneys have limited recovery capacity after AKI, which can explain why most of our patients with AKI developed renal Fanconi syndrome.

More than half of our AKI patients died shortly after AKI, with no preceding signs of multiple organ involvement. AKI is known as an important precipitant of endstage renal failure. The 5-year mortality after AKI is high even among patients treated with renal replacement therapies [18]. Our results suggest that AKI is important to recognize as it suggests decompensating mitochondrial disease and poor short-term survival outcome. Increased awareness among mitochondrial disease physicians is therefore vital, as early detection and initiation of prevention, including hydration and removing nephrotoxic drugs, are the mainstays of treatment.

AKI was the first manifestation of mitochondrial disease in 3 of our patients, either alone or in combination with other clinical signs or symptoms. Other renal manifestations in previously undiagnosed patients were proximal tubulopathy, with lactic aciduria and/or generalized aminoaciduria, renal Fanconi syndrome, arterial hypertension, and kidney dysplasia. Whilst we recognize that there are more common causes, our findings suggest that an underlying mitochondrial disease should be considered in patients with (i) proximal or generalized tubulopathy especially in the presence of lactic aciduria or generalized aminoaciduria, (ii) unexpected AKI in whom common causes are absent, or (iii) arterial hypertension in children, adolescents, or young adults in the absence of risk factors for primary hypertension. When these renal manifestations are preceded by systemic infection or other metabolic stress, occurring as part of a multiorgan involvement, or when at least 2 of the abovementioned manifestations concur, the suspicion of underlying mitochondrial dysfunction is warranted.

Renal involvement was first captured on laboratory tests, either as part of routine workup or incidentally in $57 \%$ of our patients. In half of these patients, increased creatinine and/or cystatin C levels in serum were the only sign. It is, therefore, likely that patients with mitochondrial disease, and especially those with multisystemic involvement, have renal involvement that will remain undetected unless actively sought after. Hence, prudent and preplanned monitoring of renal function should be part of routine follow-up of patients with mitochondrial disease. In our protocol for the assessment and monitoring of renal function in mitochondrial disease patients without known renal involvement, we propose a laboratory assessment of renal function yearly and upon episodes of acute metabolic decompensation, shown in Table 3. As cystatin $\mathrm{C}$ appears to have a higher diagnostic accuracy than creatinine in assessing GFR among patients with mitochondrial defects [19], it should be included in the routine follow-up. Since we show that normal renal imaging does not exclude renal disease, we propose that renal imaging is performed at diagnosis of mitochondrial disease and repeated when renal manifestations occur. Renal biopsy is an invasive procedure and should be reserved when diagnostic difficulties occur or for monitoring of specific treatments.

One limitation of this study was that, based on the definition criteria used for nephritic syndrome, mild forms of nephritic syndrome may have been missed. Another limitation was the use of different biomarkers, such as se- 
rum creatinine, serum cystatin $\mathrm{C}$, or both, to evaluate renal function. As direct measurement of GFR either by $99 \mathrm{mTc}$-DTPA (diethylenetriaminopenta-acetic acid), 51Cr-EDTA (ethylenediaminetetra acetic acid), or iohexol is complicated and expensive, GFR is usually estimated using an endogenous biomarker, most commonly serum creatinine or cystatin C, in order to calculate an eGFR using the Schwartz or other equations [20]. However, all these indirect methods to estimate GFR are vulnerable to several types of bias arising, e.g., from hyperfiltration states, patient's sex, race, diet, age (for creatinine-based estimations), various diseases such as thyroid disease, low muscle mass (for creatinine), and high-dose corticosteroid treatment (for cystatin C). It is also worth mentioning that due to lack of standardization of the 2 most common assays to measure cystatin C, in each GFR estimation equation, the same method that was used for cystatin $\mathrm{C}$ measurement in the equation should be applied [20].

Our study shows that AKI is a distinct renal phenotype in mitochondrial diseases, and that it is associated with poor disease outcome. Early and prompt recognition of AKI and of renal disease in general is crucial for optimal patient management. A protocol for the assessment of renal function is proposed to help physicians to systematically monitor their patients. Early detection of renal symptoms and signs in a mitochondrial disease patient will facilitate a better usage of the available therapeutic arsenal and improve quality of life of the patient and reduce long-term complications and treatment costs.

\section{Statement of Ethics}

Our study complied with the ethical guidelines and was conducted in accordance with the World Medical Association Declaration of Helsinki. The study protocol was approved at each par- ticipating center. As this was a retrospective study of de-identified patient data, the need for informed consent was waived by the regulatory authorities (Ethics Committee Etikprövningsmyndigheten, Sweden [previously Regionala Etikprövningsnämnden Göteborg]; METC, Rotterdam; The Ethics Committee of Northern Ostrobothnia Hospital District, Oulu; The Ethics Committee of Helsinki University Hospital, Helsinki; Regional Ethics Committee, Bergen; Ethics and Research Committee of the Fundación Sant Joan de Déu, Institut de Recerca Hospital Sant Joan de Déu, Barcelona; Danish Patients Safety Authority and the Capital Region of Denmark).

\section{Conflict of Interest Statement}

The authors have no conflicts of interest to declare.

\section{Funding Sources}

This study was supported by grants from the Swedish Research Council (ALFGBG 77820).

\section{Author Contributions}

All authors equally contributed to study conception and design, data acquisition, and critical review of the results and of the manuscript. Maria Parasyri and Kalliopi Sofou also performed data management and statistical analysis as well as drafting of the manuscript. Kalliopi Sofou and Niklas Darin share co-last authorship.

\section{Data Availability Statement}

All data generated or analyzed during this study are included in this article. Further enquiries can be directed to the corresponding author.

\section{References}

1 Darin N, Oldfors A, Moslemi AR, Holme E, Tulinius M. The incidence of mitochondrial encephalomyopathies in childhood: clinical features and morphological, biochemical, and DNA anbormalities. Ann Neurol. 2001;49(3): $377-83$.

2 Rahman S, Hall AM. Mitochondrial disease: an important cause of end-stage renal failure. Pediatr Nephrol. 2013;28(3):357-61.

3 Martín-Hernández E, García-Silva MT, Vara J, Campos Y, Cabello A, Muley R, et al. Renal pathology in children with mitochondrial diseases. Pediatr Nephrol. 2005;20(9):1299-305.
4 Neiberger RE, George JC, Perkins LA, Theriaque DW, Hutson AD, Stacpoole PW. Renal manifestations of congenital lactic acidosis. Am J Kidney Dis. 2002;39(1):12-23.

5 Pérez-Albert P, de Lucas Collantes C, Fernández-García MÁ, de Rojas T, Aparicio López C, Gutiérrez-Solana L. Mitochondrial disease in children: the nephrologist's perspective. JIMD Rep. 2018;42:61-70.

6 Niaudet P, Rotig A. The kidney in mitochondrial cytopathies. Kidney Int. 1997;51(4): $1000-7$.

7 O’Toole JF. Renal manifestations of genetic mitochondrial disease. Int J Nephrol Renovasc Dis. 2014;7:57-67.
8 Emma F, Bertini E, Salviati L, Montini G. Renal involvement in mitochondrial cytopathies. Pediatr Nephrol. 2012;27(4):539-50.

9 Bhargava P, Schnellmann RG. Mitochondrial energetics in the kidney. Nat Rev Nephrol. 2017;13(10):629-46.

10 Govers LP, Toka HR, Hariri A, Walsh SB, Bockenhauer D. Mitochondrial DNA mutations in renal disease: an overview. Pediatr Nephrol. 2021 Jan;36(1):9-17.

11 Schwartz GJ, Work DF. Measurement and estimation of GFR in children and adolescents. Clin J Am Soc Nephrol. 2009;4(11):1832-43. 
12 KDIGO 2012 clinical practice guideline for the evaluation and management of CKD. Kidney Inter Suppl. 2013;3:1-150.

13 Kdigo akiwg 2012: kidney disease: improving global outcomes (KDIGO) clinical practice guideline for acute kidney injury. Kidney Inter. 2012;2(1):1-141.

14 Igarashi T. Pediatric fanconi syndrome. In Avner E, Harmon W, Niaudet P, Yoshikawa N, Emma F, Goldstein S, editors. Pediatric Nephrology. Berlin, Heidelberg: Springer; 2014. p. 1.

15 Parikh S, Goldstein A, Karaa A, Koenig MK, Anselm I, Brunel-Guitton C, et al. Patient care standards for primary mitochondrial disease: a consensus statement from the Mitochondrial Medicine Society. Genet. Med.. 2017;19: 1380.

16 Wang Y, Zhu J, Liu Z, Shu S, Fu Y, Liu Y, et al. The PINK1/PARK2/optineurin pathway of mitophagy is activated for protection in septic acute kidney injury. Redox Biol. 2020 Feb 1;38(2):101767.
17 Tran M, Tam D, Bardia A, Bhasin M, Rowe GC, Kher A, et al. PGC-1 1 p promotes recovery after acute kidney injury during systemic inflammation in mice. J Clin Invest. 2011;121:4003-14.

18 Havasi A, Borkan SC. Apoptosis and acute kidney injury. Kidney Int. 2011;80:29-40.

19 Lee SM, Kim JH, Lee YM, Lee JS, Kim HD. Evaluation of renal function in children with mitochondrial respiratory chain complex defect: usefulness of cystatin C. Acta Paediatr. 2009;98(6):1014-8.

20 Pasala S, Carmody JB. How to use... serum creatinine, cystatin $\mathrm{C}$ and GFR serum creatinine, cystatin $\mathrm{C}$ and GFR. Arch Dis Child Educ Pract Ed. 2017 Feb;102(1):37-43.

21 Freyer C, Stranneheim H, Naess K, Mourier A, Felser A, Maffezzini C, et al. Rescue of primary ubiquinone deficiency due to a novel COQ7 defect using 2,4-dihydroxybensoic acid. J Med Genet. 2015 Nov;52(11):779-83.
22 Sofou K, Kollberg G, Holmström M, Dávila M, Darin N, Gustafsson CM, et al. Whole exome sequencing reveals mutations in NARS2 and PARS2, encoding the mitochondrial asparaginyl-tRNA synthetase and prolyl-tRNA synthetase, in patients with Alpers syndrome. Mol Genet Genomic Med. 2015 Jan;3(1):59-68

23 Komulainen T, Hautakangas MR, Hinttala R, Pakanen S, Vähäsarja V, Lehenkari $P$, et al. Mitochondrial DNA depletion and deletions in paediatric patients with neuromuscular diseases: novel phenotypes. JIMD Rep. 2015; 23:91-100.

24 O’Toole JF, Liu Y, Davis EE, Westlake CJ, Attanasio $\mathrm{M}$, Otto $\mathrm{EA}$, et al. Individuals with mutations in XPNPEP3, which encodes a mitochondrial protein, develop a nephronophthisis-like nephropathy. J Clin Invest. 2010 Mar;120(3):791-802. 